\title{
Le texte au musée pour les visiteurs non-voyants : comment aborder les choix de contenus et de formes?
}

Texts in museums for non-sighted visitors : how to approach content and format choices?

\section{Valérie Chauvey}

\section{OpenEdition}

\section{Journals}

Édition électronique

URL : http://journals.openedition.org/ocim/391

DOI : 10.4000/ocim.391

ISSN : 2108-646X

Éditeur

OCIM

Édition imprimée

Date de publication : 1 novembre 2010

Pagination : 40-47

ISSN : 0994-1908

\section{Référence électronique}

Valérie Chauvey, "Le texte au musée pour les visiteurs non-voyants : comment aborder les choix de contenus et de formes? », La Lettre de I'OCIM [En ligne], 132 I 2010, mis en ligne le 01 novembre 2012 consulté le 19 avril 2019. URL : http://journals.openedition.org/ocim/391 ; DOI : 10.4000/ocim.391

Ce document a été généré automatiquement le 19 avril 2019

Tous droits réservés 


\section{Le texte au musée pour les visiteurs non-voyants : comment aborder les choix de contenus et de formes?}

Texts in museums for non-sighted visitors : how to approach content and format choices?

\section{Valérie Chauvey}

1 Pour introduire la thématique particulière « du texte au musée pour les visiteurs nonvoyants ", je me permets d'emprunter l'image du titre du documentaire Le vent dans la boîte aux lettres, de Frédéric Batt, diffusé par Marie-José Mondzain (CNRS Paris) lors d'une de ses communications ${ }^{1}$. Le public avait découvert l'étonnant récit que le personnage principal, aveugle de naissance, faisait du bruissement occasionné par le vent soufflant sur les feuilles d'un arbre, ce qui lui permettait de localiser cet arbre et par conséquent de lui signifier son existence. Cette image inattendue et décalée du vent comme "révélateur» de présence pour les personnes voyantes que nous sommes, pourrait alors s'appliquer à certaines réflexions nées de nos recherches en muséologie sur les besoins et attentes de(s) public(s) déficient(s) visuel(s). S'agissant ici du texte dans l'exposition, la question est effectivement de savoir s'il est pensable, voire souhaitable, de donner « existence » à la quantité de textes écrits et présent(é)s dans les musées et, par conséquent, de s'interroger sur les formes que le contenu des textes expographiques choisi pourrait prendre pour être accessible aux personnes qui n'ont pas ou plus le sens de la vue pour les lire.

\section{Musées et publics enquêtés}

Dans la perspective d'apporter des réponses à ces questions précises, deux musées ont été le terrain d'enquêtes conduites auprès de personnes déficientes visuelles: le musée Hector Berlioz de La Côte-Saint-André (38) et le muséum de Lyon, redéployé en musée des 
Confluences (69). Les résultats disponibles à l'heure actuelle proviennent d'enquêtes basées sur une méthode dont les outils sont sans surprise ceux des sociologues : au sein des deux institutions muséales citées, des suivis de médiations et des observations participantes ont été réalisés entre 2005 et 2007. Des entretiens semi-directifs ont été menés auprès de personnes travaillant à l'accueil, à la médiation, à la conservation. Plus largement, des observations indirectes effectuées en accompagnant une personne aveugle ont eu lieu à Paris (le musée de l'Air et de l'Espace du Bourget, le Palais de la Découverte, le musée du Louvre, la maison de Victor Hugo, le musée du quai Branly) et à Londres (British Museum, Victoria and Albert Museum, Natural History Museum, et National Gallery). L'institution «public(s) déficient(s) visuel(s)» a été consultée dans le monde de l'enseignement spécialisé (Établissement régional d'Enseignement adapté pour Déficients de la Vue de Villeurbanne), dans la sphère associative (associations Valentin Haüy de Lyon et Grenoble, l'Amitié des Déficients visuels, de Rétina France et de la Fidev à Lyon), ou encore rencontrée lors de colloques ou de forums de discussions accessibles sur Internet. Certaines de ces personnes ont répondu à des entretiens semi-directifs, participé à des visites tests ou encore visité de nombreux musées (cités) pour expérimenter les dispositifs en situation. Enfin, un envoi de questionnaires pour connaître les pratiques muséales des personnes déficientes visuelles a été effectué par Internet à destination de personnes appartenant à ces divers réseaux associatifs ou personnels cités plus haut. Cent cinquante sept personnes ont répondu au questionnaire dont les résultats ont été traités et analysés à l'aide du logiciel Sphinx.

\section{Sur les musées et les publics déficients visuels}

3 Les constatations d'ordre général sur la présence de dispositifs d'accessibilité dans les musées sont liées d'une part aux modes de vie qu'entraîne le positionnement du musée dans son territoire. Les conditions d'accès à un musée situé en zone rurale ne sont pas identiques à celles d'un musée de zone urbaine par exemple (un arrêt de tramway est prévu aux portes du futur musée des Confluences, tandis que les moyens de transport en commun entre Grenoble et La Côte-Saint-André sont quasiment inexistants). D'autre part, l'efficacité des dispositifs tient également à la présence active d'associations ou d'équipements d'enseignement spécialisé ou de structures professionnelles dans le handicap concerné sur ce même territoire (on compte 23 associations spécialisées dans la déficience visuelle dans le bassin lyonnais, couvrant tous les âges de la vie). Ainsi, la synergie réelle entre le(s) public(s) déficient(s) visuel(s) et les personnes chargées de la médiation (ou du service des publics quand le musée en est doté) permet une offre culturelle considérable. «À Lyon, on a la culture de la visite au musée » tient à rappeler une personne aveugle fortement impliquée dans l'association lyonnaise «Le musée du bout des doigts $»^{2}$.

\section{Le texte au musée}

Quand le visiteur est bien voyant, l'appropriation du lieu muséal et de l'exposition s'effectue par la découverte du parcours de visite pensé par les concepteurs, en cheminant au gré des espaces, en détaillant du regard des objets phare exposés. Cette « liberté » de déplacement dans l'espace se retrouve également dans la manière de lire les textes expographiques comme les cartels ou les panneaux. Le sens de la vue permet à 
l'individu qui voit, de découvrir le contenu des mots en même temps que leur forme et ainsi d'en décrypter les codes de lecture (charte graphique, identité visuelle). Ces éléments visuels sont les outils utilisés par les concepteurs qui souhaitent donner sens à leurs intentions et guider le visiteur dans la découverte de ce discours, et que MarieSylvie Poli appelle «la main courante " ${ }^{3}$. Les personnes lisent autant les textes expographiques (une affiche, un panneau) que la mise en exposition (la vue d'ensemble, l'esthétique, l'identité visuelle). Nous pouvons même dire qu'un visiteur voyant «lit» l'exposition de la même manière qu'il lit un texte imprimé : le contenu apparaît en même temps que les mises en évidence typographiques ou spatiales qui lui sont attribuées, et le regard offre une compréhension globale du lieu quasiment instantanément par effet d'apprentissage, d'habitude et de pratique. Cette manière de signifier un contenu par la forme typographique n'existe que pour les textes imprimés qu'on appelle aussi « en noir » (comme celui-là même que vous lisez en ce moment) : dans un lieu comme un musée, notre regard averti peut lire successivement des textes de contenus très hétérogènes, comme par exemple un panneau à fonction signalétique puis à contenu informatif de type cartel ou panneau. Beaucoup de textes se retrouvent ainsi lus de manière passive ou spontanée. Ces textes en noir offrent également la possibilité à plusieurs personnes de lire de manière synchrone leur contenu: chaque lecteur peut y trouver de l'information selon la situation, ses capacités au moment de la lecture (conditions de visite) et ses intérêts.

5 La personne qui ne voit pas, ou plus, accède de manière autre au monde qui l'entoure. Les éléments de l'espace global échappent, et seul l'espace à portée de main s'appréhende de manière circonscrite et séquentielle ${ }^{4}$ : de manière auditive ou tactile, des détails sont perçus un à un, les uns après les autres. Il est important de souligner que si une information tactile peut être prise et renouvelée par le toucher, les sons quand à eux ne peuvent être captés qu'une seule fois. Pour qu'une personne aveugle puisse construire une «image mentale " d'ensemble de ces éléments disparates, il lui faut du temps, de l'énergie, de la concentration, ainsi que le sentiment d'être en confiance et en sécurité. Certains éléments lointains ou proches ne prennent «vie » qu'avec l'intervention d'une personne qui les voit et qui les décrit. Ainsi, l'accès à toutes les informations (formes et contenus) concernant un lieu comme un musée n'est pas pensable à l'heure actuelle, et le choix des informations à rendre accessibles doit faire l'objet d'une réflexion réunissant en groupe de travail des acteurs comme les équipes des musées (direction, service des publics, médiateurs, service de communication), des universitaires, et des personnes représentant les publics à besoins spécifiques pour valider les dispositifs à l'usage. Les élus politiques locaux doivent être également sensibilisés à la mise en accessibilité des transports en commun (annonces vocales dans les bus, sites Internet...).

\section{Dispositifs spécifiques d'accès au texte}

6 L'écriture braille (1829) et le logiciel de synthèse vocale adapté à tout ordinateur classique (1985) sont les deux inventions techniques qui ont révolutionné l'accès au texte pour les personnes aveugles et malvoyantes.

7 Peut-être faut-il dire qu'aujourd'hui, environ 10000 aveugles complets lisent le braille en France ${ }^{5}$ : toutes les personnes aveugles ne sont donc pas braillistes contrairement à ce que nous croyons. Ajoutons également qu'un support écrit en braille dans un lieu public suppose un unique lecteur et des niveaux de lecture différents (contrairement, comme 
nous l'avons vu plus haut, au texte en noir qui permet plusieurs lecteurs et plusieurs niveaux de lecture). Si l'usage du braille pour certaines personnes braillistes permet d'acquérir de la connaissance par l'accès direct à la lecture sans intermédiaire humain ou technique et l'accès à l'orthographe des mots, pour beaucoup d'autres personnes non braillistes, le braille représente une barrière infranchissable de par la difficulté de son apprentissage (surtout pour les personnes ayant perdu la vue tardivement et/ou ayant le bout des doigts abîmés), de la longueur de certains textes qui nécessitent de la concentration et procurent une grande fatigue cognitive. Des supports de texte en braille proposés à des personnes aveugles pratiquant peu ou pas le braille peuvent alors occasionner un effet stigmatisant sur ces personnes et provoquer parfois un repli sur elles-mêmes.

8 Les utilisateurs d'ordinateurs adaptés, quant à eux, dépassent de $10 \%$ la population nationale dite " ordinaire » équipée en matériel informatique. Nathalie Lewi Dumont disait en 2005 qu'il faudrait idéalement créer un livre par personne malvoyante tant les déficiences visuelles sont nombreuses et diverses ${ }^{6}$. Les adaptations techniques actuelles pour accéder à l'information écrite apportent de réelles solutions et respectent les compétences de chaque individu déficient visuel lecteur d'écran : parmi ces adaptations à la malvoyance, on peut citer les plus essentielles, comme le télé agrandisseur qui grossit l'affichage de l'écran et facilite la visualisation des textes, images, icônes ou dossiers. Les choix de contrastes permettent également de mettre un texte en lettres blanches sur un fond noir et inversement. Pour les adaptations à la cécité totale, le scanner, la machine à lire et enfin l'ordinateur doté d'un logiciel de voix synthétique permettent l'accès aux textes imprimés ou numériques.

9 La synthèse vocale a pour autre fonction de rendre sonores les informations jusque-là visuelles pour guider l'utilisateur dans la navigation de l'environnement de l'ordinateur. Cette voix de synthèse peut être masculine ou féminine, de même qu'elle est réglable en fréquence constante (sans intonation) et en volume, en fonction du contenu du texte à lire et de la situation de lecture. L'observation de personnes déficientes visuelles utilisatrices d'ordinateur à synthèse vocale nous apprend que tous ces réglages de vitesse et de fréquence rendent possible le «survol » d'un texte. Le rendu « robotisé » de la voix ne gêne aucunement les personnes déficientes visuelles pour qui l'accès à l'information prime sur la forme. Par contre, la plupart des personnes voyantes qui entendent pour la première fois cette voix synthétique de l'ordinateur adaptée en mode « débit rapide et atone » disent non seulement ne rien comprendre de ce qui est « dit » mais surtout jugent désagréable cette voix robotisée. Cependant, si la voix synthétique est appropriée pour rendre un contenu informatif, de repérage ou de travail, elle peut devenir plus humaine pour aborder une forme littéraire: les ouvrages contemporains, vendus en format " audio » sont d'ailleurs de plus en plus enregistrés par la voix de leurs propres auteurs. Il existe également des associations qui demandent à de nombreux bénévoles de "prêter " ou «donner» leur voix à de nombreux ouvrages. Un appareil lecteur-enregistreur embarqué permet également la prise de notes ${ }^{7}$ par la fonction dictaphone, et la « lecture " par l'écoute de nombreux livres audio selon la capacité de la carte mémoire choisie. Nous pouvons donc retenir que si les codes de lecture «audio » diffèrent des codes de lecture visuelle, l'accès au(x) texte(s) dans les diverses modalités de lecture cidessus citées est aujourd'hui possible, pour qui est équipé et formé aux logiciels informatiques bien évidemment. 


\section{Dispositifs spécifiques de médiation}

10 De nombreux dispositifs sont maintenant mis en place dans le(s) musée(s) pour les personnes aveugles et malvoyantes: des visites guidées, des visites tactiles, avec parfois des supports thermogonflés ou maquettes bi ou tri dimensionnelles, ou même des cartels permanents en braille et gros caractères, ou encore cahiers embarqués écrits en double lecture (noir et braille). Pour répondre plus précisément aux attentes d'information sur l'espace global du lieu, les dispositifs observés au cours de l'exposition Frontières du muséum de Lyon en 2005, consistaient à faire commencer le parcours par une conférence dans la salle audiovisuelle du muséum, et de continuer la visite guidée à l'aide de cartes de géographie thermogonflées. Au musée Hector Berlioz, nous avons enregistré sur mp3 une interview de l'assistant de conservation sous forme de conversation, insistant sur les choix de la muséographie, sur les étapes successives de la déambulation dans les étages, tout cela sur fond sonore de parquets qui craquent et d'anecdotes sur la vie du musicien ou sur des objets présent(é)s dans la maison. Cet enregistrement a été donné à écouter, en amont d'une visite test, à plusieurs personnes aveugles. Il a été constaté que l'écoute de cet enregistrement permettait au futur visiteur de se faire une idée topographique du lieu mais aussi de se créer un horizon d'attentes: la description de certaines pièces, de certains objets comme des bustes de Berlioz ou même le tabouret du piano en forme de coquillage, mais également le rendu sonore comme l'écho des pas sur les parquets en sapin différent sur les parquets en chêne selon que la pièce est un salon ou une chambre, ou même des extraits de morceaux musicaux enregistrés dans l'auditorium ont suscité de la curiosité et de l'intérêt que seuls le déplacement géographique et la visite de la maisonmusée pouvaient satisfaire. Dans le même temps, la retranscription de l'enregistrement et la numérisation du texte enregistré ont été mis à disposition de personnes déficientes visuelles afin d'en permettre la lecture multiple selon les techniques disponibles à chacun (braille, gros caractère, synthèse vocale) respectant ainsi les capacités de lecture individuelles de chaque lecteur. À l'usage, c'est-à-dire une fois le groupe constitué au départ de Grenoble pour aller visiter le musée de La Côte-Saint-André, il a été observé que ce dispositif textuel «lu» en amont de la visite par les visiteurs déficients visuels a facilité non seulement la compréhension du lieu in situ mais a permis pour certaines personnes de se construire une place dans l'espace social: durant le déplacement en voiture, ces personnes disposant d'informations sur Berlioz et sa maison natale ont pu tout simplement parler de leurs lectures et contribuer à l'échange discursif avec les autres participants.

\section{L'enquête par questionnaires}

11 Comme il a été dit plus haut, un questionnaire a été conçu pour mieux connaître les habitudes muséales des personnes aveugles et malvoyantes. Cent cinquante sept personnes vivant en France ont répondu par mél ou par courrier ; elles se répartissent de la manière suivante : femmes ( $49 \%)$ et hommes $(51 \%)$; plus de 55 ans $(27,4 \%)$, entre 35 et 55 ans $(42 \%)$, entre 25 et 35 ans (19,7\%), entre 18 et 25 ans (12\%); mariés ou compagnon $(55,4) \%)$, célibataires, veufs ou divorcés $(36,3 \%)$; malvoyants et très malvoyants $(36,2 \%)$; aveugles tardifs $(44,6 \%)$; aveugles de naissance $(17,2 \%)$; utilisateurs de canne blanche 
(59,2\%), utilisateurs de chien guide $(15,3 \%)$; avec activité professionnelle ( $45 \%)$, sans activité professionnelle (32,5\%), retraités (12\%), étudiants (6\%).

Reposant principalement sur des questions ouvertes, les réponses au questionnaire ont apporté de nombreux commentaires inattendus.

\section{L'interdiction de toucher en question}

13 À la question "aimez-vous aller au musée ", $55,4 \%$ des personnes ont répondu par l'affirmative, pourcentage auquel nous pouvons ajouter les 15,3\% qui ont répondu aimer aller au musée «si le musée est adapté ». Selon la majorité de ces témoignages, l'incontournable injonction de «ne pas toucher » caractériserait encore le monde muséal. Mais il semblerait que ce constat puisse être mis en équilibre avec les réponses faites à la question «Pourquoi avez-vous aimé ce musée ?» : en effet, 29 personnes (soit 18,5\%) parlent d'expériences vécues comme " exceptionnelles ", "rares » et qui témoignent au contraire de la souplesse que certains personnels de musées montrent vis-à-vis de la fameuse « interdiction de toucher»:

- le musée du Louvre : «Un ami voyant avait organisé une visite adaptée un mardi (jour de fermeture au public), avec un groupe de 15 handicapés moteurs et visuels. Guide très agréable, détaillant bien les œuvres sans excès et permission de toucher les objets les moins fragiles. Nous étions seuls dans les galeries, le Louvre était à nous, nous ne gênions pas les autres visiteurs et inversement... »;

- le musée Grévin : «Parce que j’ai eu droit à une visite guidée du début jusqu'à la fin, par un gardien fort sympathique, qui m'avait disputée parce que je touchais le Général de Gaulle. Comme j'étais de dos, il ne savait pas que j'étais non-voyante. Lorsque je me suis retournée et expliqué pourquoi je touchais, alors il m'a proposé une visite. J'ai ainsi eu accès à toutes les scènes même celles de la Révolution française, et j'ai passé une après-midi extraordinaire, mais trop rare ».

Ne pourrions-nous pas établir un lien entre la forte émotion "ressentie» par ces personnes et cette souplesse «dite» sur l'institution autorisant de toucher "l'intouchable »? Un médiateur lors d'une visite tactile a réellement insisté sur le côté " privilégié » de ces médiations. Le témoignage qui suit donne l'occasion de réfléchir aux conséquences éventuelles d'une institutionnalisation de cette pratique: «Un musée Zadkine vers Cahors, qui fut une victoire personnelle : l'agent d'accueil m'a d'abord refusé l'autorisation de toucher, puis voyant qu'elle avait peu d'autres clients, elle est revenue sur sa décision et Zadkine a fait l'émerveillement. L'autre musée Zadkine avant qu'il ne soit adapté aux aveugles : j'ai touché absolument à toutes les œuvres trois heures de bonheur épuisant. Quand j'y suis retourné, $20 \%$ des œuvres seulement étaient autorisées... ».

\section{Compagnon de visite : rôle et interaction}

Si 82 personnes disent qu'aller au musée «c'est compliqué » $(52,2 \%)$ parce qu'il faut trouver «obligatoirement» une personne qui les accompagne, 56 autres personnes répondent qu'« aller au musée est simple » (35,7\%), si elles sont accompagnées dans leur déplacement avant d'arriver au musée et pendant le parcours de visite muséale. Ainsi, au total, presque $89 \%$ des personnes disent ne pas souhaiter se rendre seules dans un musée. 
Ce résultat, d'une part, se rapproche des chiffres donnés par l'enquête du Centre de Recherche pour l'Étude et l'observation des conditions de vie, menée en juin $2005^{8}$, avec $86 \%$ des personnes qui déclarent aller au musée accompagnées; mais d'autre part, contredit le discours entendu de nombreuses fois dans les colloques sur la nécessité de faire venir seules au musée les personnes handicapées: "Aveugle, oui! Spéléologue, non! ».

Si des personnes accompagnatrices sécurisent les personnes aveugles et malvoyantes par leur présence dans la découverte du lieu et le parcours de visite, elles donnent des informations sur l'espace global et lisent également les textes expographiques 9 L'interaction entre le visiteur et son compagnon en fait donc une relation privilégiée. Une anecdote vécue au musée de la maison de Victor Hugo en 2005 nous a permis de constater que la médiation au départ conçue pour faire participer les personnes aveugles avait complètement omis le rôle du compagnon de visite et avait eu pour conséquence de rompre l'interaction entre le visiteur et son compagnon : la médiation consistait en effet à reconstruire l'univers littéraire de l'écrivain comme une enquête policière, par une série d'indices à découvrir. Lors d'une de ces séquences mises en scène, alors que les personnes aveugles et compagnons de visite étaient assis autour de la même table, l'indice à trouver était un personnage célèbre d'un roman de Victor Hugo, personnage représenté par une sculpture, disposée sur la table, que la personne aveugle percevait, touchait en même temps qu'elle s'assurait auprès de son compagnon de visite que ce qu'elle touchait était bien une étoffe, un visage, ou tout autre élément important à la découverte du personnage sculpté. Il s'avère que le nom de ce personnage était gravé sur le socle de la sculpture et que le médiateur n'avait pas pris soin de le dissimuler. Les lettres du surnom de «Cosette » se sont donc imposées par leur visibilité à mon regard. Ayant pris connaissance de cet indice contre ma volonté et sans effort, j'ai éprouvé un vif sentiment de malaise vis-à-vis de la personne que j'accompagnais, malaise qui s'est incarné par un silence rompant net ainsi l'interaction.

\section{Implications des conditions de visite dans les souvenirs}

17 Des traces laissées par une visite au musée, 11,5\% des personnes disent ne pas avoir de mauvais souvenir. Selon les témoignages concernant les expériences négatives $(20,4 \%)$, les premières raisons évoquées portent sur l'inaccessibilité en général, « l'interdiction de toucher ", "la présence de vitrines », ou encore « une mauvaise organisation interne au musée » : « Aucune possibilité de toucher et il fallait d'abord payer avant de savoir si les non voyants pouvaient bénéficier d'une quelconque aide!» (musée d'Art moderne de New York).

Pour $12,1 \%$ des réponses, les mauvais souvenirs tiennent de la thématique de l'exposition, comme la peinture ou l'art moderne souvent cités comme étant «trop abstrait » :

- un musée à Montauban : «Avec mon père et ma sœur aînée qui y passaient des heures. Je n'ai aucune idée de la peinture qui reste abstraite pour moi ».

- un musée à Florence : «Je faisais partie d'un voyage professionnel, où tous les autres étaient voyants, et se régalaient à regarder des salles et des salles de tableaux. Au début ils essayaient de me raconter, et très vite j'en ai eu marre, car ce n'était pas très parlant. Je leur 
ai donc demandé de m'asseoir sur une chaise dans un coin et de me récupérer lorsqu'ils auraient fini. Ce qu'ils ont fait ».

19 Puis sont citées les conditions de la visite (11,5\%), comme le confort en général, qui peut concerner l'accompagnement, la longueur de la visite, le mauvais éclairage, ou le bruit prodigué par la foule: "J'apprécie moins les gros musées avec beaucoup de monde et dont la visite est interminable; trop de choses à voir sature l'esprit de celui qui ne voit pas ".

Le contexte individuel de la situation peut également porter ombre au bon déroulement de la visite $(6,4 \%)$ : "J'ai ouvert la porte des toilettes au lieu d'une porte de salle...", « c'était bruyant, peu d'explication et mon chien avait perdu sa clochette... ».

Pour en revenir au "texte au musée ", un témoignage écrit par une personne aveugle dans l'un de ces questionnaires relate qu'un agent à l'accueil d'un musée lui a remis un document en braille, document qui n'avait «aucun intérêt, puisqu'il s'agissait de la description des moyens de transports pour arriver au musée ».

\section{Conclusion}

Imaginer une visite au musée pour personnes qui ne voient pas peut sembler étrange aux personnes voyantes que nous sommes. Les témoignages de ces enquêtes prouvent qu'on ne fait pas que voir au musée : les personnes aveugles et malvoyantes vont au musée pour se cultiver (93\% des réponses du questionnaire pour personnes déficientes visuelles) au même titre que les personnes voyantes ( $89 \%$ des personnes interrogées dans l'enquête CREDOC). L'intérêt porté aux pratiques muséales des personnes aveugles et malvoyantes permet de soulever des questions plus spécifiques mais aussi plus larges que l'aspect de la visite muséale suppose en elle-même : des questions liées au territoire et à l'implantation du musée dans celui-ci, induisant alors les questions liées au déplacement dans l'espace urbain ou rural et à l'accessibilité matérielle des bâtiments, mais aussi des questions liées aux motivations de l'institution muséale dans sa politique d'offre culturelle et des moyens humains mis en place (connaissance des besoins spécifiques de certains publics, offre de formation aux personnels d'accueil, accès à la connaissance des dispositifs d'accès au texte et supports ou médiations spécifiques).

Mais ces questions qui traitent de l' « accessibilité » rejoignent les questions sous jacentes à la démocratisation culturelle : les personnes aveugles et malvoyantes qui ont répondu à l'enquête par questionnaire réunissent les conditions optimales pour avoir des activités culturelles : une activité professionnelle, un conjoint, une activité associative, mais aussi l'autonomie des déplacements dans l'espace urbain. Si ces personnes qui vont au musée entre deux et cinq fois $(34,4 \%)$ dans l'année pensent que cela est « compliqué », que fautil en déduire pour les autres personnes qui n'y vont pas? Les personnes vieillissantes, vivant seules, sans famille ou avec un faible réseau amical ou associatif ou résidant en institution restent privées de contact avec l'extérieur, et par conséquence avec la culture. La culture s'adresserait-elle encore aux plus privilégiés, même dans le monde du handicap ? ${ }^{10}$ Le contact familiarisé avec les personnes aveugles, la recherche théorique mais également la présence sur le terrain permettent alors d'avoir des réponses décalées avec ce qu'il est aujourd'hui proposé dans certains musées ou entendu dans certains discours : comprendre pourquoi en tant que visiteurs, les personnes aveugles éprouvent la nécessité 
d'anticiper et de programmer une visite au musée, parce qu'il leur faut trouver un compagnon de visite et un musée avec dispositifs, ou de chercher les conditions favorisant au maximum leur sécurité plus que leur autonomie, préférant pour certains des visites les jours de fermeture des musées ou la visite en groupe de personnes déficientes visuelles; comprendre pourquoi, par exemple, le braille n'est pas le moyen le plus « évident » comme support d'accès au texte à proposer sans pour autant l'exclure... L'étude des pratiques muséales de ces visiteurs à besoins spécifiques nous amène à conclure que la visite au musée représente une expérience sociale et individuelle qui rassemble alors tous les visiteurs sous une même entité, celle d'un public qui vit cette expérience dans son esprit mais dans son corps: une phrase tirée de cette enquête par questionnaire le dit mieux que quiconque: «Ce n'est pas moi qui entre dans le musée mais le musée qui entre en moi ».

\section{NOTES}

1. La peinture dans le noir, Bruxelles, juin 2006, colloque organisé par La Ligue Braille en partenariat avec les musées royaux des Beaux-Arts de Belgique.

2. "Le musée du bout des doigts " propose un cycle annuel d'une dizaine de visites au musée pour les personnes aveugles et malvoyantes (visites thématiques avec découverte tactile d'œuvres et description de tableaux, supports pédagogiques en relief).

3. Poli, M.-S. L'écrit au musée : comment les dispositifs de textes informatifs sont-ils perçus par les visiteurs? Cahiers de recherche de l'École doctorale en linguistique française, p. 21.

4. Hatwell, Y., Streri, A. et Gentaz, É. Toucher pour connaître, psychologie cognitive de la perception tactile manuelle. PUF, 2000.

5. Journées d'Études Littérature de jeunesse et déficience visuelle, Cnefei. Suresnes, 2005.

6. La population en situation de handicap visuel en France. Importance, caractéristiques, incapacités fonctionnelles et difficultés sociales : une exploitation des enquêtes HID 1998 et 1999. Observatoire régional de la santé des Pays de la Loire, juillet 2005

7. La prise de notes en braille peut être également faite à l'aide d'une tablette et d'un poinçon : la personne brailliste fixe une feuille de papier épais dans une tablette quadrillée de cases correspondant aux six points de l'alphabet braille et enfonce le poinçon dans les cases correspondant aux lettres. Les points enfoncés dans la feuille épaisse de droite à gauche deviennent alors saillants quand la feuille est retirée de la tablette et retournée pour être lue de gauche à droite.

8. Enquête du CREDOC (Centre de Recherche pour l'Etude et l'Observation des conditions de vie) menée en juin 2005 sur la "Fréquentation et image des musées", commande de la Direction des Musées de France, 2000 entretiens face à face.

9. Il est intéressant de montrer ici comment les résultats de notre enquête s'inversent avec ceux de l'enquête du CREDOC : les compagnons privilégiés de visite pour les déficients visuels sont d'abord «les amis » à 44,6\%, avant « le conjoint » à 14,6\%. Le groupe enquêté par le CREDOC répond d'abord « le conjoint » à $74 \%$ et ensuite « les amis » à $39 \%$. Il semble que les personnes aveugles et malvoyantes souhaitent soulager la charge que leur conjoint assume au quotidien : la lecture des textes expographiques demande au compagnon un grand investissement cognitif, et 
les amis sont donc mis à contribution pour garder l'aide du conjoint «pour ce qui est le plus important ».

10. Pratiques muséales de personnes aveugles ou malvoyantes et dispositifs d'accessibilité à l'épreuve de l'usage, 15 avril 2010, colloque organisé par le Museo tattile statale Omero (Ancone, Italie) «L'accessibilità al patrimonio museale e leducazione artistica ed estetica delle persone con minorazione visiva ».

\section{RÉSUMÉS}

Faut-il présenter au public malvoyant la totalité des textes contenus dans les expositions? Quelles formes cette présentation peut-elle revêtir ? Une enquête menée dans des musées et au sein du réseau associatif travaillant dans le domaine de l'aide aux déficients visuels permet d'apporter quelques éléments de réponse à ces interrogations.

Does one need to present to partially and non-sighted visitors all content texts in exhibitions? What format can this presentation take ? A survey carried out in museums, and within the association network working in the field of aid to people with impaired vision, endeavours to answer some of these questions.

INDEX

Mots-clés : étude de publics, exposition, handicap, non-voyants, réseau, texte, visiteur

\section{AUTEUR}

\section{VALÉRIE CHAUVEY}

Valérie Chauvey est doctorante à l'université Pierre Mendès France de Grenoble. valeriechauvey@free.fr 